\title{
Widespread subdural metastasis from breast cancer progressing rapidly with cerebral herniation: A case report
}

\author{
YOSHIKO OKITA ${ }^{1}$, NORIKAZU MASUDA ${ }^{2}$, MAKIKO MIZUTANI $^{2}$, YOSHINORI KODAMA $^{3,4}$, \\ KIYOSHI MORI ${ }^{3}$, MASAYUKI MANO ${ }^{3}$, TOMOYOSHI NAKAGAWA ${ }^{1}$, \\ SHIN NAKAJIMA ${ }^{1}$ and TOSHIYUKI FUJINAKA ${ }^{1}$ \\ Departments of ${ }^{1}$ Neurosurgery, ${ }^{2}$ Surgery, Breast Oncology and ${ }^{3}$ Central Laboratory and Surgical Pathology, \\ National Hospital Organization Osaka National Hospital, Osaka 540-0006; ${ }^{4}$ Department of Pathology \\ and Applied Neurobiology, Kyoto Prefectural University of Medicine, Kyoto 602-8566, Japan
}

Received January 31, 2017; Accepted March 17, 2017

DOI: $10.3892 / \mathrm{mco} .2017 .1233$

\begin{abstract}
We herein present the case of a 49-year-old female patient presenting with nausea and headache. The patient's medical history included breast cancer with bone and lymph node metastasis. Computed tomography (CT) examination revealed a high-density lesion in the right subdural space, suggesting hematoma. During surgery planned for subdural hematoma drainage, an en plaque subdural yellowish-white tumor was identified, without hematoma. Histopathological examination revealed metastatic breast cancer. The patient was administered predonisolone and her neurological symptoms gradually recovered. However, 12 days after the first operation, the clinical course was complicated by vomiting and rapid loss of consciousness. Emergency CT revealed that the subdural tumor had enlarged and decompression was performed as life-saving surgery. However, the patient's condition progressively deteriorated and she finally succumbed to the disease 2 months after the second operation. The aim of this study was to present the case of a patient with a large en plaque subdural tumor mimicking subdural hematoma and causing rapid loss of consciousness and cerebral herniation.
\end{abstract}

\section{Introduction}

Dural metastasis from extraneural malignancies is rare, having been detected during autopsy in $10 \%$ of the cases of extraneural malignancies (1). Breast cancer is one of the tumors that frequently metastasizes to the dura (2), whereas dural metastasis with subdural hematoma is infrequently reported (3-5). By contrast, Nayak et al reported that $25 \%$ of dural metastases,

Correspondence to: Dr Yoshiko Okita, Department of Neurosurgery, National Hospital Organization Osaka National Hospital, 14-1-2, Hoenzaka, Chuo-ku, Osaka 540-0006, Japan E-mail: yokita4246@gmail.com

Key words: subdural metastasis, breast cancer, cerebral herniation in which the most common primary site was breast cancer, exhibited diffuse dural involvement (6). However, only few reports detail large en plaque subdural tumor without hematoma in breast cancer (7). We herein report the case of a patient with breast cancer with a widespread en plaque subdural tumor, which mimicked subdural hematoma and caused rapid loss of consciousness and cerebral herniation.

\section{Case report}

A 49-year-old woman was admitted to the National Hospital Organization Osaka National Hospital (Osaka, Japan) with nausea and headache. The patient had previously undergone resection, radiotherapy and chemotherapy for bilateral breast cancer. Local control of the primary lesion was maintained, but multiple systemic metastasis to the bones and lymph nodes developed. Computed tomography (CT) revealed a high-density lesion in the right subdural space, suggesting hematoma. Midline shift to the left was observed (Fig. 1A and B). Enhanced CT showed no parenchymal brain lesions. A high-density lesion in the right subdural space was homogeneously enhanced (Fig. 1C and D). As a tissue expander had been implanted in the patient's breast, magnetic resonance imaging (MRI) could not be performed.

A right frontal burr hole was drilled for subdural hematoma drainage. Upon opening the dura mater, a yellowish-white subdural tumor was identified, without evidence of old or fresh hemorrhage. A biopsy was performed and the histopathological examination of the tissue revealed metastatic breast cancer. The patient received $40 \mathrm{mg} /$ day prednisolone and the neurological symptoms gradually subsided. However, 12 days after the first operation, the clinical course was complicated by vomiting and rapid loss of consciousness. An emergency $\mathrm{CT}$ examination revealed that the subdural tumor had enlarged and the midline shift to the left was exacerbated. The subdural tumor also caused uncal herniation, with upper brainstem compression (Fig. 2A). A fronto-temporo-parietal craniotomy for decompression was performed as life-saving surgery. Direct inspection indicated that the outer membrane of the dura and the inner side of the skull were not breached by the tumor. The dura was opened and an en plaque fronto-temporo-parietal subdural yellowish-white 

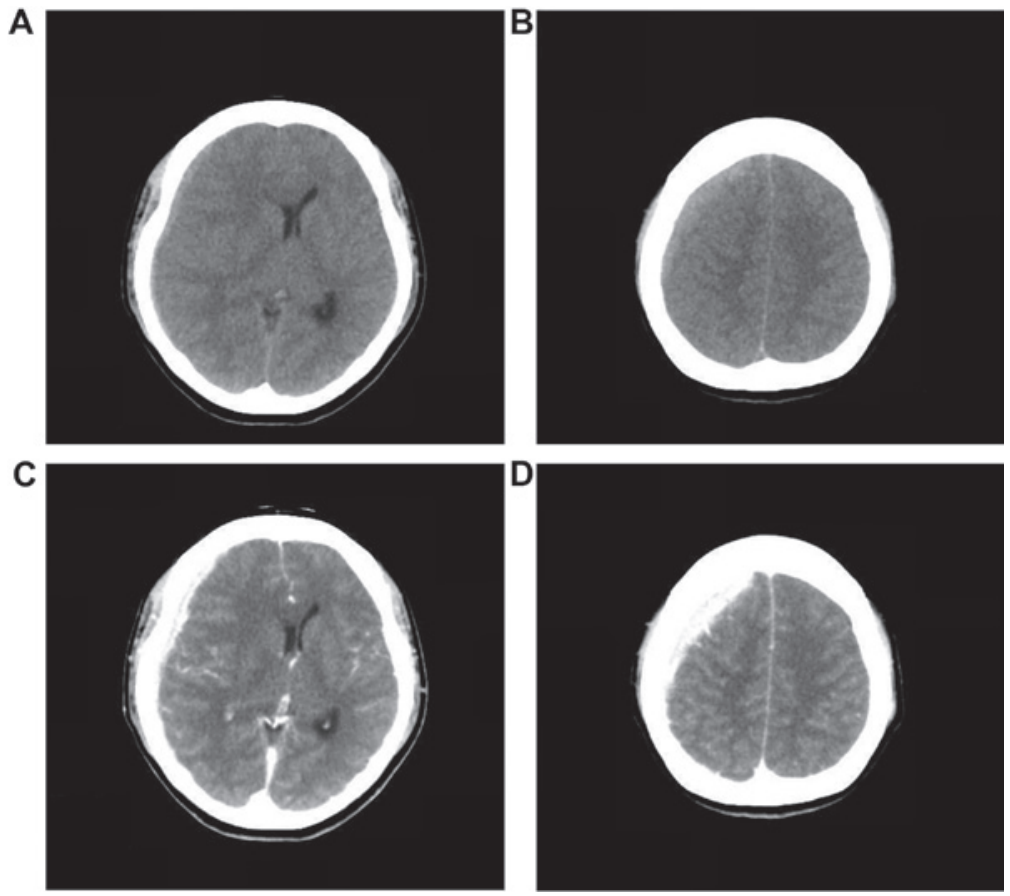

Figure 1. (A and B) Computed tomography (CT) images showing a high-density lesion in the right subdural space, suggestive of a hematoma. Midline shift to the left was also observed. (C and D) Enhanced CT showed no parenchymal brain lesions. A high-density lesion in the right subdural space was homogeneously enhanced.

A

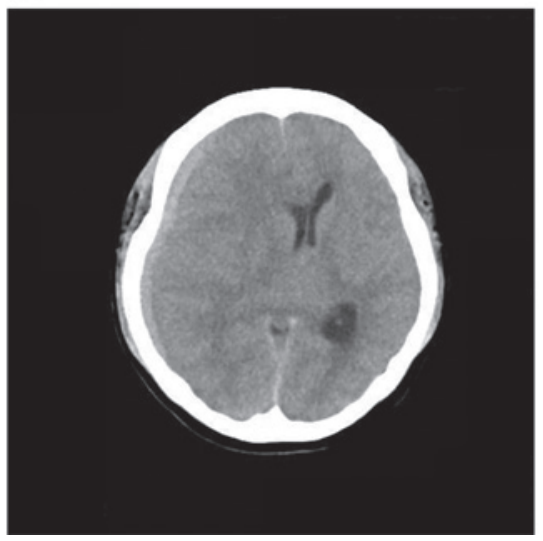

B

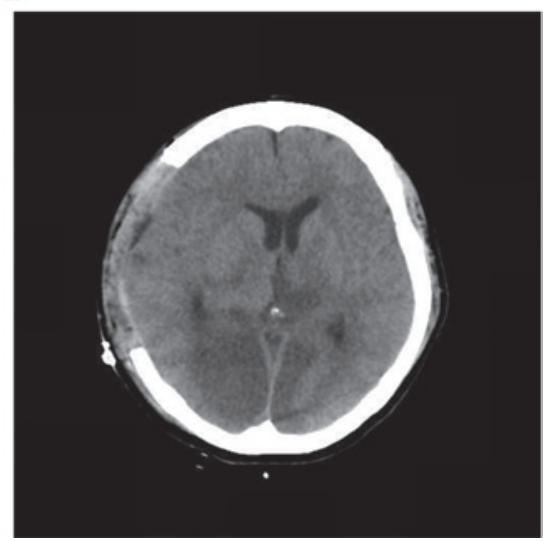

Figure 2. (A) Emergency computed tomography (CT) revealed that the subdural tumor had enlarged and the midline shift to the left was exacerbated. The subdural tumor had also caused uncal herniation with upper brainstem compression. (B) The CT revealed bilateral posterior cerebral artery infarct due to cerebral herniation.

tumor was identified in the subdural space, with no cortical involvement, but with involvement of the adjacent dura.

Unfortunately, even post-surgery, the patient's consciousness failed to improve and a CT revealed bilateral posterior cerebral artery infarct due to the cerebral herniation (Fig. 2B). The patient's condition progressively deteriorated and she finally succumbed to the disease 2 months after the second operation.

\section{Discussion}

Dural metastasis is now more frequently diagnosed owing to advanced radiological imaging techniques. Dural metastasis from extraneural malignancies has been detected in $\sim 10 \%$ of autopsy cases (1). In a series of 198 cases of dural metastasis, the tumors most commonly metastasizing to the dura mater were cancer of the prostate $(19.5 \%)$, breast $(16.5 \%)$, lung (11\%) and stomach (7.5\%) (2). Tsukada et al reported that the more common clinically diagnosed central nervous system metastatic patterns in breast cancer were metastasis to the brain alone $(25.0 \%)$, a combination of brain and cranial dura metastasis $(15.6 \%)$, and metastasis to the cranial dura alone $(13.5 \%)(8)$.

Nzokou et al identified three image patterns in dural metastasis: i) A nodule in a subdural hematoma, ii) multinodular metastasis surrounded by a subdural hematoma and iii) an extensive en plaque subdural tumor (9). In cases of extensive en plaque subdural tumors, where unexpectedly no blood was 
found, a larger craniotomy revealed an extensive tumor not amenable to surgical treatment (9). The characteristics of our case conformed to the latter type, namely a large en plaque subdural tumor. In a previous report, $25 \%$ of dural metastases, in which the most common primary site of was breast cancer, exhibited diffuse dural involvement (6). However, only few reports have detailed widespread en plaque subdural tumors in breast cancer, and the prognosis in the reported cases was poor (7). Neurological symptoms of dural metastasis are due to irritation of the dura by the tumor, intracranial edema and cerebral herniation (10). As the brain is enclosed in a rigid container, within which there is little room for expansion, a widespread en plaque subdural tumor may cause rapid loss of consciousness and cerebral herniation, which was the case in our patient. Consequently, life-saving decompression was warranted.

Dural metastasis has been reported to mimic subdural hematoma (7,11-13). The operative method appropriate for metastatic subdural tumor with midline shift may differ completely from that for chronic subdural hematoma. In cases of en plaque subdural tumors, the preoperatively planned burr hole or craniotomy must be converted into a larger craniotomy (9). If preoperative diagnosis is difficult, the operative procedures must be adjusted. Contrast-enhanced CT is appropriate for an emergency operation and should reveal a homogeneously enhanced, thickened dura in cases of subdural metastasis (11). In the present case, contrast-enhanced CT revealed a homogeneously enhanced lesion in the dura, representing a metastasis. Therefore, clinicians should bear in mind the possibility of not only brain parenchymal, but also dural metastasis from primary breast cancer.

\section{Acknowledgements}

The present study was supported by Grant-in-Aid for Scientific Research from the Ministry of Education, Science and Culture of Japan (no. 16K20033 to Y.O.).

\section{References}

1. Meyer PC and Reah TG: Secondary neoplasms of the central nervous system and meninges. Br J Cancer 7: 438-448, 1953.

2. Laigle-Donadey F, Taillibert S, Mokhtari K, Hildebrand J and Delattre JY: Dural metastases. J Neurooncol 75: 57-61, 2005.

3. Kunii N, Morita A, Yoshikawa G and Kirino T: Subdural hematoma associated with dural metastasis-case report. Neurol Med Chir (Tokyo) 45: 519-522, 2005.

4. Otsuka A, Asakura K, Takahashi K, Tasaki T, Okada K and Suzuki Y: Nontraumatic chronic subdural hematoma due to dural metastases of breast cancer. Case report. No Shinkei Geka 13: 999-1004, 1985 (In Japanese).

5. Tseng SH, Liao CC, Lin SM, Chen Y and Shun CT: Dural metastasis in patients with malignant neoplasm and chronic subdural hematoma. Acta Neurol Scand 108: 43-46, 2003.

6. Nayak L, Abrey LE and Iwamoto FM: Intracranial dural metastases. Cancer 115: 1947-1953, 2009.

7. Villano JL and Ryan CW: Patients presenting with CNS lesions. Case 2. Subdural presentation of recurrent breast cancer. J Clin Oncol 21: 4060-4062, 2003.

8. Tsukada Y, Fouad A, Pickren JW and Lane WW: Central nervous system metastasis from breast carcinoma. Autopsy study. Cancer 52: 2349-2354, 1983.

9. Nzokou A, Magro E, Guilbert F, Fournier JY and Bojanowski MW: Subdural metastasis of prostate cancer. J Neurol Surg Rep 76: e123-e127, 2015.

10. Posner JB and Chernik NL: Intracranial metastases from systemic cancer. Adv Neurol 19: 579-592, 1978.

11. Cheng YK, Wang TC, Yang JT, Lee MH and Su CH: Dural metastasis from prostatic adenocarcinoma mimicking chronic subdural hematoma. J Clin Neurosci 16: 1084-1086, 2009.

12. Patil S, Veron A, Hosseini P, Bates R, Brown B, Guthikonda B and DeSouza R: Metastatic prostate cancer mimicking chronic subdural hematoma: A case report and review of the literature. J La State Med Soc 162: 203-205, 2010.

13. Tomlin JM and Alleyne CH: Transdural metastasis from adenocarcinoma of the prostate mimicking subdural hematoma: Case report. Surg Neurol 58: 329-331, 2002. 